\title{
Desove espontáneo, ontogenia y crecimiento en cautiverio de Cynoscion squamipinnis (Perciformes: Sciaenidae)
}

\author{
Jorge Boza-Abarca, Marvin Ramírez-Alvarado, Juan Barquero-Chanto, Emilia Calvo-Vargas \& \\ Karen Berrocal-Artavia \\ Estación de Biología Marina Juan Bertoglia Richards, Escuela de Ciencias Biológicas, Universidad Nacional, \\ Puntarenas, Costa Rica; jorge.boza.abarca@una.cr, marvin.ramirez.alvarado@una.cr, juanes0489@gmail.com, \\ emilia.calvo.vargas@una.cr, karen.berrocal.artavia@una.cr
}

\section{Recibido 10-XI-2015. C Corregido 04-III-2016. Aceptado 04-IV-2016.}

\begin{abstract}
Spontaneous spawning, ontogeny and growth in captivity of Cynoscion squamipinnis (Perciformes: Sciaenidae). The croakers or drums are commercial species, which have been overfished in the Nicoya Gulf, Costa Rica. This study aimed to describe, for the first time, the reproduction and the ontogeny of weakfish, Cynoscion squamipinnis in captivity, in order to perform restocking and mariculture projects. Wild fish $(n=6$, 1-2 Kg) were captured and maintained in the Estación de Biología Marina Juan Bertoglia Richards (Puntarenas, Costa Rica) for a two years period (October 2006- December 2008). During this period, maturation stage was monitored periodically by cannula samples in the females $(n=3)$ and gentle massage in males $(n=3)$. All fish were stocked in an $18 \mathrm{t}$ tank, with aeration, 33-35 ups of salinity, and a constant temperature $\left(29 \pm 1{ }^{\circ} \mathrm{C}\right)$. The spawning period occurred from January to March 2009, producing 162000 eggs in three spontaneous spawns. The fertilization percentage was 50-60\%, and survival after hatching was $60-85 \%$. The egg diameter was 0.852 $\mathrm{mm}$ (Standard deviation $(\mathrm{SD})=0.039)$, and oil drop of $0.269 \mathrm{~mm}(\mathrm{SD}=0.016)$. In the embryonary development, the first mitotic division (MD) was observed one hour after spawning (has), the second MD was 1:30 has, the third MD was 2:00 has, the fourth MD was 2:30 has, and fifth MD at 3:00 has. Morule was observed 3:30 has, the blastule 4:30 has, the gastrule 8:30 has, $\mathrm{C}$ shape at 10:00 has, and $\mathrm{C}$ shape at 12:00 has. After 19 has hatching larvae occurred. The total length (TL) of the larvae was $2.234 \mathrm{~mm}(\mathrm{SD}=0.122)$, and the nothochordial length $(\mathrm{NL})$ was $2.179 \mathrm{~mm}(\mathrm{SD}=0.119)$. Preflexion stage was observed 49 has, flexion stage was 11 days after spawn (das) (3.767 mm LT $(\mathrm{SD}=0.209))$, and postflexion stage was 14 das $(4.015 \mathrm{~mm} \mathrm{LT}(\mathrm{SD}=0.302))$. After 45 das, the juvenile weights $3.68 \mathrm{~g}(\mathrm{SD}=1.09)$. Hatch time of the weakfish larvae was minor than of others croaker species. The stages times of embrionary development were a little different from others croaker species, and probably respond to genetic characteristics of each species and the eggs incubation temperature. The spontaneously spawning without broodstock hormonal applications, and the juveniles production in captivity showed that weakfish is a potential species for restocking programs and mariculture projects. Rev. Biol. Trop. 64 (3): 991-1005. Epub 2016 September 01.
\end{abstract}

Key words: Cynoscion squamipinnis, weakfish, reproduction, ontogeny, rearing larvae.

La corvina aguada, Cynoscion squamipinnis, es una de las especies de mayor valor comercial en el golfo de Nicoya, y junto con la corvina reina, Cynoscion albus, representan más del $50 \%$ de la captura pesquera en la costa pacífica de Costa Rica (Chacón et al., 2007). Estas corvinas forman parte de las 34 especies que se encuentran distribuidas en la costa pacífica de América, y de los 70 géneros (270 especies) en todo el mundo (Thomas, Arnold, \& Holt, 1995; Cárdenas, 2012).

Actualmente, el interés por el cultivo de corvinas en el mundo se ha incrementado debido a la fuerte presión pesquera (Jiménez et al., 2005). En el caso de la corvina aguada (C. squamipinnis), su captura con trasmallo 
varió de 166 TM en 2002 a 258 TM en 2003, llegando a su valor más bajo reportado de 142 TM en 2005 (Chacón et al., 2007).

Algunas especies de esciánidos se han estudiado con el interés de repoblar áreas que han sido sobreexplotadas, mientras que otras especies han sido estudiadas por su potencial en la acuicultura (Cárdenas, 2011; Duncan et al., 2012; Thomas et al., 1995), siendo alrededor de 18 especies las criadas en el mundo (Cárdenas, 2012). La sobreexplotación de las poblaciones naturales de peces ha ocasionado que la acuicultura adquiera mayor importancia, y se tome en cuenta como una alternativa empresarial y alimenticia (Otárola, 2008; FAO, 2012). Aún así, el estudio del ciclo reproductivo de estos peces en Centroamérica es muy limitado (Ditty, Shaw, \& Farooqui, 2006). Esta es la situación en la que se encuentra la corvina aguada, de ahí el interés en controlar su reproducción y el alevinaje en cautiverio, para desarrollar proyectos de maricultura.

En estudios recientes, se ha determinado que las corvinas alcanzan el desarrollo gonadal en cautiverio, sin embargo, necesitan la inducción al desove mediante tratamientos hormonales (Cárdenas, 2012; Duncan et al., 2012; Mylonas, Mitrizakis, Papadaki, \& Sidelaki, 2013a; Mylonas et al., 2013b), como inyecciones de hCG (García-Alonso \& Vizziano, 2004), o inyecciones e implantes de GnRH (Cárdenas, 2011; 2012; Duncan et al., 2012). Según Jiménez et al. (2005) y Cárdenas (2011), la única especie con desoves espontáneos en cautiverio es el corvinón ocelado (Sciaenops ocellatus).

Además, el levantamiento larval se caracteriza por altas mortalidades que se presentan en las diferentes etapas de desarrollo. Esta mortalidad se debe a diversos factores, destacándose la calidad nutricional del alimento vivo y su tamaño (Ignatius, Rathore, Jagadis, Kandasami, \& Victor, 2001), así como el manejo de la temperatura y la salinidad (Fielder, Bardsley, Allan, \& Pankhurst, 2005). Por lo anterior, el conocimiento sobre el desarrollo embrionario es uno de los aspectos más importantes para tener éxito en la producción acuícola de especies de peces marinos de interés comercial (Botta et al., 2010; Hazmadi et al., 2013).

En la corvina mediterránea (Argyrosomus regius), Jiménez, Rodrígues, de La Rúa, Sánches, \& Cárdenas (2007) y Gamsiz y Neke (2008) describieron los cambios que experimentan los huevos y las larvas recién eclosionadas hasta completar la forma de adulto pequeño 30 días después del desove (ddd), al igual que Ballagh, Pankhurst, \& Fielder (2011) con la especie Argyrosomus japonicus. Koumoundouros et al. (2005), estudiaron el desarrollo ontogénico del verrugato (Umbrina cirrosa), al clasificarla como una especie candidata para la acuicultura. En otras especies marinas, BozaAbarca, Calvo-Vargas, Solís-Ortiz, \& Komen (2008), realizaron la descripción del desarrollo larval de Lutjanus guttatus, mientras que Cuartas, Rosas, Velásquez, \& Cabrera (2003) lo realizaron para Haemulon bonariensis, y Hernández, Perera, Castillo, \& Luna (2012), para la especie Eugerres mexicanus.

Al considerar la importancia ecológica y pesquera de la corvina aguada en Costa Rica, el objetivo del presente estudio fue describir por primera vez la reproducción en cautiverio y el desarrollo embrionario y larval de la corvina aguada, C. squamipinnis, para contribuir con el conocimiento de estas especies, evaluar su reproducción en cautiverio y la factibilidad de producir juveniles para un futuro repoblamiento o proyectos de maricultura.

\section{MATERIAL Y MÉTODOS}

En octubre 2006, las corvinas (C. squamipinnis) ( $\mathrm{n}=6$, tres hembras y tres machos), fueron colectadas en la parte interna del Golfo de Nicoya por medio de línea taiwanesa, para ser utilizadas como reproductores. Los peces con un peso entre $1-2 \mathrm{Kg}(1.80 \pm 0.47 \mathrm{Kg}$, promedio $\pm \mathrm{DE}$ ) y longitud total de $40-50 \mathrm{~cm}$ $(45.0 \pm 3.8 \mathrm{~cm}$, promedio $\pm \mathrm{DE})$, fueron mantenidos en la Estación de Biología Marina Juan Bertoglia Richards de la Universidad Nacional, Puntarenas, Costa Rica, en un tanque cilíndrico de fibra de vidrio a una densidad de 0.3 peces/ $\mathrm{m} 3$. Los peces fueron alimentados diariamente 
con raciones de sardina picada ( $2 \%$ peso corporal) durante dos años, y cada mes, el estado de madurez fue observado, mediante la canulación (hembras) y masaje abdominal (machos) (Boza-Abarca et al., 2008).

El tanque de desove consistió de un recipiente cilíndrico de fibra de vidrio de $18 \mathrm{t}$, adaptado a las condiciones de desove (cubierta de sarán $80 \%$ sombra), aireación constante (20 psi), con colector de huevos con malla fina $(250-500 \mu \mathrm{m})$, y recambio de agua diario $(80$ $\%)$. El recolector de huevos consistió de un contenedor de fibra de vidrio cubierto de malla fina menor a las $500 \mu \mathrm{m}$ (volumen de $20 \mathrm{~L}$ ). El momento del desove fue registrado mediante la presencia de huevos en la superficie del tanque o del recolector.

La salinidad (ups) fue medida con un refractómetro SR5-E, la temperatura $\left({ }^{\circ} \mathrm{C}\right)$ y el oxígeno disuelto $(\mathrm{mg} / \mathrm{L})$ fueron medidos con un multiparámetros YSI DO200, tanto en el tanque de desove, como en los tanques de larvas.

Los huevos en el tanque de desove fueron recolectados por rebalse, y se estimó el número total y el porcentaje de fertilización. Los huevos fueron limpiados bajo las mínimas condiciones asépticas (recambios de agua irradiada (30 000 $\mathrm{mW} / \mathrm{sec} . \mathrm{cm} 2$ ), retiro de impurezas), luego se colocaron en un contenedor de $2 \mathrm{~L}$, del cual se extrajeron muestras de $1 \mathrm{~mL}(\mathrm{n}=3)$, que fueron colocadas independientemente en cajas de petri cuadriculadas, para luego realizar los conteos de la cantidad de huevos presentes, y se obtuvo un promedio por desove. Después del conteo de los huevos, éstos fueron colocados en tanques cilíndricos de fibra de vidrio de $1 \mathrm{t}$, con $500 \mathrm{~L}$ de agua de mar filtrada (poro malla $10 \mu \mathrm{m}$ ) e irradiada (UV). La temperatura en los tanques de larvas fue entre $26-30{ }^{\circ} \mathrm{C}\left(27.11 \pm 0.72{ }^{\circ} \mathrm{C}\right.$, promedio $\pm \mathrm{DE}$ ), la salinidad entre $32-35$ ups (33.52 \pm 0.79 ups), y el oxígeno entre $5-6 \mathrm{mg} / \mathrm{L}$ $(5.40 \pm 0.50 \mathrm{mg} / \mathrm{L})$.

Una cubierta de sarán ( $80 \%$ de sombra) se instaló para evitar la incidencia directa de la luz solar. Una aireación leve (20 psi) fue suministrada por un soplador eléctrico. Los tanques fueron inoculados con el alga Isochrysis galba$n a\left(6 \times 10^{6} \mathrm{cel} / \mathrm{mL}\right.$, día 1 al día 7$)$, y el rotífero
Brachionus plicatilis (Talla S, 20 ind/mL, día 3 al día 10). El día 11 se inició la alimentación con nauplios de Artemia (10 nauplios/ $\mathrm{mL}$, hasta el día 22), y adultos de artemia (5 adultos/mL, del día 22 al día 30). Las raciones diarias de rotíferos y artemia fueron repartidas en tres tiempos. La alimentación con nauplios de Artemia terminó el día 31, ya que la larva aceptó la alimentación con sardina fresca a partir del día 25. La transición de alimento vivo a alimento inerte se realizó combinando alimento semiseco $(20 \%)$ con sardina fresca $(80 \%)$. La proporción fue cambiando hasta que únicamente se alimentó con alimento seco (Olivares \& Boza, 1999). Todos los días se limpió el fondo de los tanques de larvas por sifoneado $(20 \%$ de recambio de agua).

Durante la fase embrionaria, se midieron el diámetro de los huevos $(\mu \mathrm{m})$ y de la gota de aceite $(\mu \mathrm{m})$ cada 6 horas $(n=30)$. Una vez que las larvas eclosionaron, todos los días se tomaron muestras de éstas $(n=30)$, y se les midió la longitud total (LT, $\mathrm{mm}$ ), la longitud notocordial (LN, mm), el diámetro de la gota de aceite (GA, mm), y se estimó el volumen del saco vitelino $\left(\mathrm{V}, \mathrm{mm}^{3}\right)$, según Ramírez-Merlano et al. (2010):

$$
\mathrm{V}=(\Pi / 6) * \mathrm{~L}^{*} \mathrm{H}^{2}
$$

donde:

$\mathrm{V}$ : volumen del saco vitelino, expresado en $\mathrm{mm}^{3}$.

L: Longitud total del saco vitelino expresado en $\mathrm{mm}$.

$\mathrm{H}$ : Altura del saco vitelino expresado en $\mathrm{mm}$.

En la fase larval se midió la longitud total (LT, mm), y la longitud notocordial (LN, mm, $\mathrm{n}=30$ ). Todas las mediciones fueron realizadas utilizando un estereoscopio MEIJI EMZ-TR, con micrómetro $( \pm 0.01 \mathrm{~mm})$. La supervivencia de las larvas (días), fue determinada después de cada ciclo ( $\mathrm{n}=3$, alrededor de 35 días). $\mathrm{Al}$ final del periodo los juveniles obtenidos fueron pesados con una balanza semi analítica $( \pm 0.01$ g).

La descripción de los estadíos embrionarios y larvales se realizaron basados en 
el registro fotográfico diario y los estudios de Moretti, Fernández, Cittolin, \& Guidastri (1999), Boza-Abarca et al. (2008), Ballagh et al. (2011), Zavala-Leal, Dumas-Lampage, \& Peña-Martínez (2011), Hernández et al. (2012), y Papadakis, Kentouri, Divanach, \& Mylonas (2013).

\section{RESULTADOS}

Después de mantener en cautiverio machos $(n=3)$ y hembras $(n=3)$ de corvina aguada, C. squamipinnis, en óptimas condiciones y durante dos años (octubre 2006 a diciembre 2008, peso entre los $8-10 \mathrm{Kg}$ ) y bajo un régimen constante de temperatura $\left(29{ }^{\circ} \mathrm{C} \pm 1\right.$ ${ }^{\circ} \mathrm{C}$ ), se inició un desove espontáneo serial, el primero que se reporta para la corvina aguada silvestre mantenida en cautiverio. En diciembre 2008, a las hembras se les tomó una muestra ovárica, detectándose ovocitos con diámetros superiores a $600 \mu \mathrm{m}$, y a los machos se les practicó un masaje abdominal con liberación de esperma. Los tres desoves se dieron en condiciones similares de laboratorio (Cuadro 1) y produjeron 162000 huevos. En el tanque de desove la salinidad fue de 33-35 ups, y la temperatura de $28-30{ }^{\circ} \mathrm{C}$. El porcentaje de fertilización fue entre el 50-60\%, y la supervivencia después de la eclosión fue entre 60-85 $\%$. Estos tres desoves fueron utilizados para la descripción del desarrollo larval de la corvina aguada $C$. squamipinnis.

Los huevos fueron concentrados y se separaron los fertilizados de los no fertilizados. Los huevos fertilizados fueron transparentes, esféricos, pelágicos y no adheribles, con un espacio perivitelino muy pequeño (Fig. 1A), un diámetro de $0.852 \pm 0.039 \mathrm{~mm}$ (promedio $\pm \mathrm{DE}, \mathrm{n}=$ 30 ), con un diámetro máximo de $0.920 \mathrm{~mm}$ y el mínimo de $0.757 \mathrm{~mm}$. La gota de lípidos midió $0.269 \pm 0.016 \mathrm{~mm}$ (promedio $\pm \mathrm{DE}, \mathrm{n}=30$ ).

La primera división mitótica se observó 1 hdd (hora después del desove, Fig. 1B), la segunda división mitótica ocurrió 1:30 hdd (Fig. 1C). A las 2 hdd las células se dividieron de nuevo dando origen a 8 células (Fig. 1D), luego a 16 células a las 2:30 hdd (cuarta división mitótica, Fig. 1E), y 32 células a las 3:00 hdd (quinta división mitótica, Fig. 1F). A las 3:30 hdd, se observó la mórula temprana (Fig. 2A), y una hora después la blástula temprana (4:30 hdd, Fig. 2B). A las 8:30 hdd se observó la gástrula (Fig. 2C), que finalizó con la formación del embrión en forma de " $\mathrm{C}$ " a las 10 hdd (Fig. 2D y Fig. 2E).

TABLE 1

Total eggs, percentage of fertilization $(\%)$, salinity (psu), temperature $\left({ }^{\circ} \mathrm{C}\right)$ and tides $($ time $(\mathrm{h})$ and highes $(\mathrm{cm}))$, and survival percentage during a serial spontaneous spawning of corvina aguada, $C$. squamipinnis

\section{CUADRO 1}

Huevos totales, porcentaje de fertilización (\%), salinidad (ups), temperatura $\left({ }^{\circ} \mathrm{C}\right)$ y mareas (tiempo (h) y Alturas (cm)), y porcentaje de supervivencia durante el desove serial espontáneo de la corvina aguada, C. squamipinnis

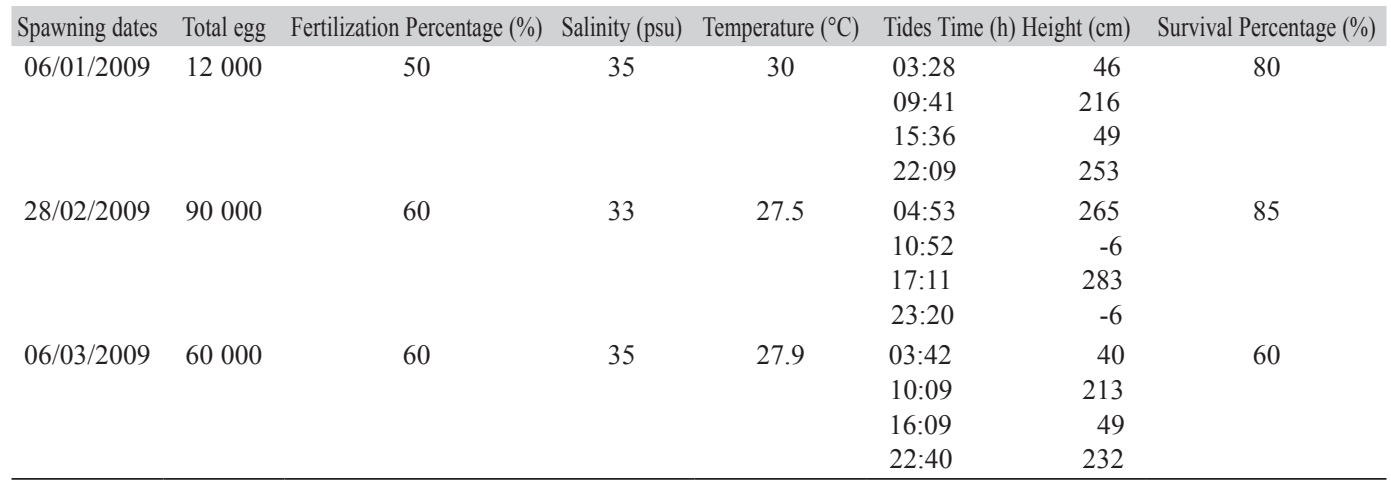




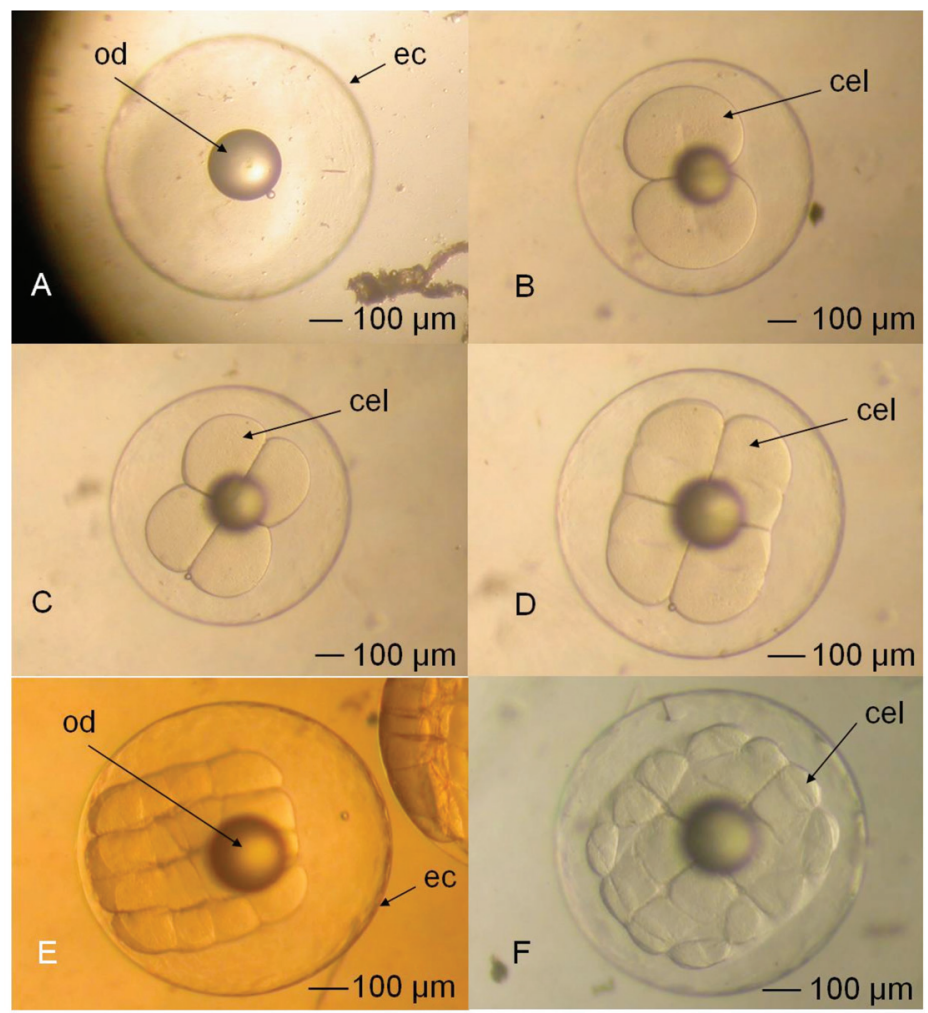

Fig. 1. Embryonic development of C. squamipinnis. (A) Fertilized egg (30 mas). (B) First mitotic division (1 has). (C) Second mitotic division (1:30 has). (D) Third mitotic division (2 has). (E) Fourth mitotic division (2:30 has). (F) Fifth mitotic division (3:00 has). (od) Oil drop. (ec) Egg capsule. (cel) Cell.

Fig. 1. Desarrollo embriónico de C. squamipinnis. (A) Huevo fertilizado (30 mdd). (B) Primera división mitótica (1 hdd). (C) Segunda división mitótica (1:30 hdd). (D) Tercera división mitótica (2 hdd). (E) Cuarta división mitótica (2:30 hdd). (F) Quinta división mitótica (3:00 hdd). (od) Gota de aceite. (ec) Cápsula de huevo. (cel) Célula.

La forma embrionaria S (Fig. 2F), ocurrió durante un periodo más extenso (10 hdd hasta las 19 hdd), caracterizada por la liberación de la cola del saco vitelino (ys), y la pigmentación de la gota de aceite (od). Fue característico encontrar espacios perivitelinos (ps), ya que el embrión (e) se observó realizando movimientos con su cola (t) para romper la cápsula del huevo.

Después de 19 hdd se observaron las primeras larvas eclosionadas (Fig. 3A), con una longitud total (LT) de $2.234 \pm 0.122 \mathrm{~mm}$ (promedio $\pm \mathrm{DE}, \mathrm{n}=30$ ), y una longitud notocordial $(\mathrm{LN})$ de $2.179 \pm 0.119 \mathrm{~mm}$ (promedio $\pm \mathrm{DE}, \mathrm{n}$ $=30$ ). La gota de aceite (od), cuyo diámetro fue de $0.269 \pm 0.016 \mathrm{~mm}$ (promedio $\pm \mathrm{DE}, \mathrm{n}=30$ ), fue visible en una posición posterior-ventral, cercana al poro anal (a) y dentro del saco vitelino (ys), el cual se extendió ventralmente hacia la cabeza. La aleta embrionaria (ef) se observó alrededor del notocordio (n).

El volumen promedio del saco vitelino (ys) al inicio fue de $0.174 \pm 0.003 \mathrm{~mm} 3 \mathrm{y}$ disminuyó hasta $0.017 \pm 0.003 \mathrm{~mm} 3,12$ hdd. Dicha disminución ocurrió progresivamente a medida que el embrión se fue desarrollando. Después de 37 hdd, el ys se absorbió (Fig. 3B), no así la od $(0.105 \pm 0.010 \mathrm{~mm}$ (promedio \pm $\mathrm{DE}), \mathrm{n}=30$ ), el ojo (e) se pigmentó y aparecieron las zonas pigmentadas (pz) alrededor del n; el tracto digestivo (dt) se engrosó y se observó a punto de abrirse. Los paquetes musculares (mp) se observaron más definidos. 


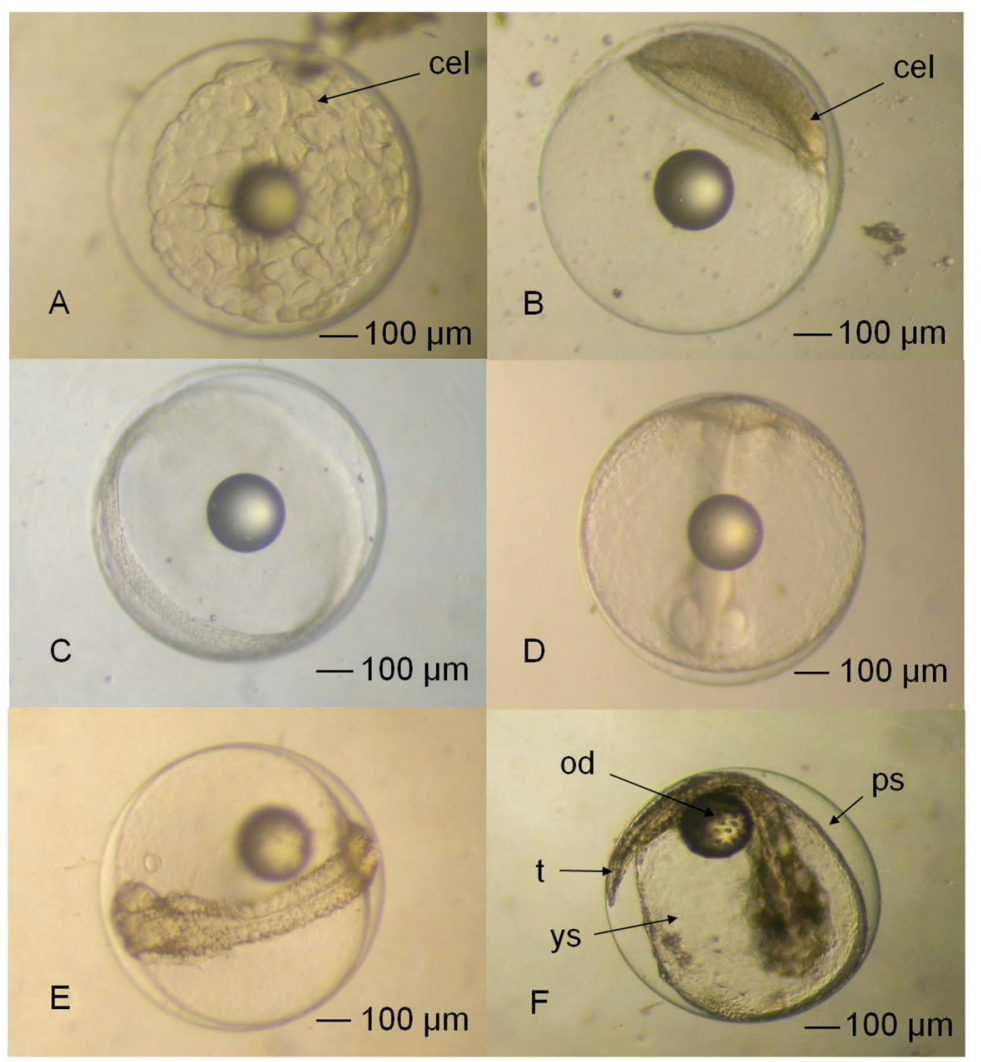

Fig. 2. Embryonic development of $C$. squamipinnis. (A) Early Morule (3:30 has). (B) Blastule (4:30 has). (C) Gastrule (8:30 has). (D) C shape embryon (10 has). (E) C shape embryon (10 has). (F) S shape embryon (12 has). (od) Oil drop. (cel) Cell. (t) Tail. (ps) Perivitelin space. (ys) Yolk sac.

Fig. 2. Desarrollo embriónico de C. squamipinnis. (A) Mórula temprana (3:30 hdd). (B) Blástula (4:30 hdd). (C) Gástrula (8:30 hdd). (D) Embrión en forma de C (10 hdd). (E) Embrión en forma de C (10 hdd). (F) Embrión en forma de S (12 hdd). (od) Gota de aceite. (cel) Célula. (t) Cola. (ps) Espacio perivitelino. (ys) Saco vitelino.

Después de 49 hdd la larva midió en promedio $2.549 \pm 0.091 \mathrm{~mm}(\mathrm{LT})$ y $2.413 \pm 0.111$ $\mathrm{mm}(\mathrm{LN})$; se inició el periodo de preflexión con la absorción total del saco vitelino, y la apertura de la boca. A los 3 días después del desove (ddd) (Fig. 3C), la larva midió $2.596 \pm$ $0.058 \mathrm{~mm}(\mathrm{LT})$ y $2.466 \pm 0.048 \mathrm{~mm}(\mathrm{LN})$, se observó un vestigio de la gota de aceite (od), la aparición de los otolitos (ot), la apertura del tracto digestivo por el ano (a), y la concentración de pigmento en la parte superior del tracto digestivo (pi) y en la cola (pz). A los 4 ddd, la larva midió en promedio $2.705 \pm 0.069 \mathrm{~mm}$ (LT) y $2.566 \pm 0.087 \mathrm{~mm}(\mathrm{LN})$ y se desarrolló la vejiga natatoria (sb) (Fig. 3D).
A los 10 ddd (3.612 $\pm 0.215 \mathrm{~mm}$ (LT) y $3.429 \pm 0.217 \mathrm{~mm}(\mathrm{LN})$ ), Fig. 3E), la cavidad visceral se pigmentó y aparecieron los dientes. Todavía persistió la aleta embrionaria, se notaron los primordios de las aletas dorsal (dfp), anal (afp), y caudal (cfp), cuya aparición indicó el final del periodo de preflexión, y el inicio del periodo de flexión. A los 13 ddd (Fig. 3F) $(3.860 \pm 0.253 \mathrm{~mm}(\mathrm{LT})$ y $3.635 \pm 0.233 \mathrm{~mm}$ (LN)) ocurrió la reducción de la aleta embrionaria y la aparición de los radios en las aletas anal (afr) y caudal (cfr). La vejiga urinaria (ub) se encontró cercana del ano.

La postflexión ocurrió 14 ddd (Fig. 4A, $4.015 \pm 0.302 \mathrm{~mm}(\mathrm{LT})$ y $3.768 \pm 0.209 \mathrm{~mm}$ 

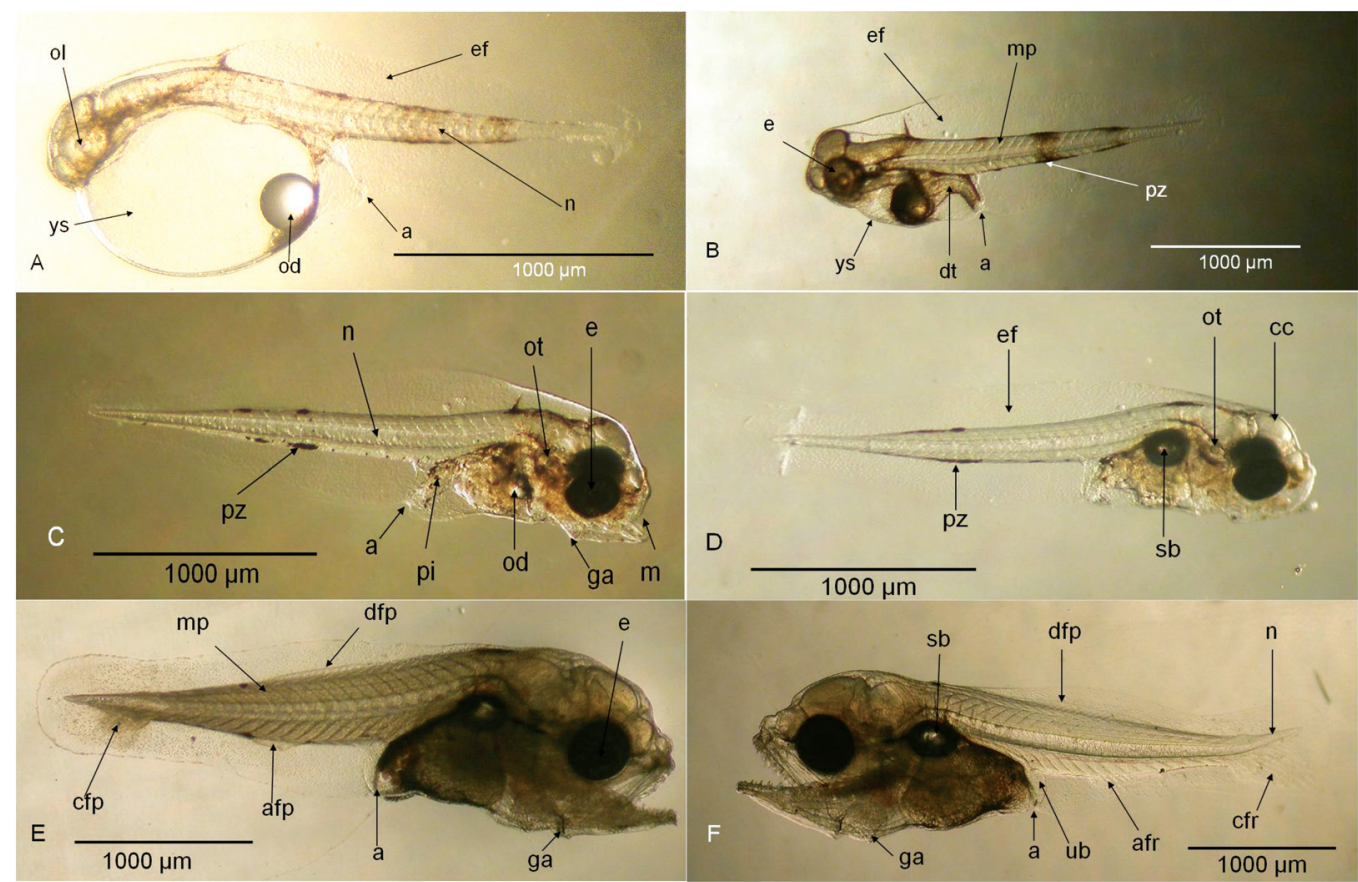

Fig. 3. Larval development of C. squamipinnis. (A) Hatched larvae (19 has). (B) Absorption of vitelin sac (37 has). (C) Open mouth larvae (3 das). (D) Swim bladder inflation larvae (4 das). (E) Preflexion larvae (10 das). (F) Flexion larvae (11 das). (ol) Ocular len. (ef) Embryonary fin. (n) Notochord. (a) Anus. (od) Oil drop. (ys) Yolk sac. (e) Eye. (dt) Digestive tract. (pz) Pigmented zone. (mp) muscular package. (ot) Otolith. (m) Mouth. (ga) Gill archers. (pi) pigmented intestine. (cc) cerebral capsule. (sb) Swim bladder. (dfp) Dorsal fin primordium. (cfp) Caudal fin primordium. (afp) Anal fin primordium. (cfr) Caudal fin radius. (ub) Urinary bladder. (afr) Anal fin radius.

Fig. 3. Desarrollo larval de C. squamipinnis. (A) Larva eclosionada (19 hdd). (B) Absorción del saco vitelino (37 hdd). (C) Larva con la boca abierta (3 ddd). (D) Larva con la vejiga natatoria llena (4 ddd). (E) Larva en preflexión (10 ddd). (F) Larva en flexión (11 ddd). (ol) Lente ocular. (ef) Aleta embrionaria. (n) Notocordia. (a) Ano. (od) Gota de aceite. (ys) Saco vitelino. (e) Ojo. (dt) Tracto digestivo. (pz) Zona pigmentada. (mp) Paquete muscular. (ot) Otolito. (m) Boca. (ga) Arcos branquiales. (pi) Intestino pigmentado. (cc) Cápsula cerebral. (sb) Vejiga natatoria. (dfp) Primordio de la aleta dorsal. (cfp) Primordio de la aleta caudal. (afp) Primordio de la aleta anal. (cfr) Radios de la aleta caudal. (ub) Vejiga urinaria. (afr) Radios de la aleta anal.

(LN)), se observó la formación de la columna vertebral (sc) y la determinación morfológica de las aletas dorsal (df), y caudal (cf), en las cuales fueron fácilmente distinguibles los radios, no así las espinas. En el caso de la aleta caudal, ésta adquirió la forma característica de punta de lanza, que fue diferente de la observada durante la fase anterior (flexión), la cual fue redondeada.

A los 16 ddd, se completó la forma de la aleta anal (af), con la formación de los radios, los arcos branquiales se encontraron cubiertos por el opérculo (o), y numerosos dientes se distribuyeron alrededor de la boca. El proceso de postflexión continuo con la osificación y formación de las vértebras de la columna (17 ddd, $4.705 \pm 0.832 \mathrm{~mm}(\mathrm{LT})$ y $3.767 \pm 0.204$ $\mathrm{mm}(\mathrm{LN})$ ), distinguiéndose la forma del centro de las vértebras $(\mathrm{sc})$ y las espinas neurales más osificadas en la figura 4B (20 ddd, $6.236 \pm$ $0.786 \mathrm{~mm}(\mathrm{LT})$ y $5.040 \pm 0.488 \mathrm{~mm}(\mathrm{LN})$ ).

A los 22 ddd (6.582 $\pm 0.952 \mathrm{~mm}$ (LT), $5.289 \pm 0.644 \mathrm{~mm}(\mathrm{LN})$, Fig. 4C), se observaron las aletas pélvicas (pf), y el opérculo cubriendo completamente las branquias; las espinas de la aleta anal se distinguieron de 


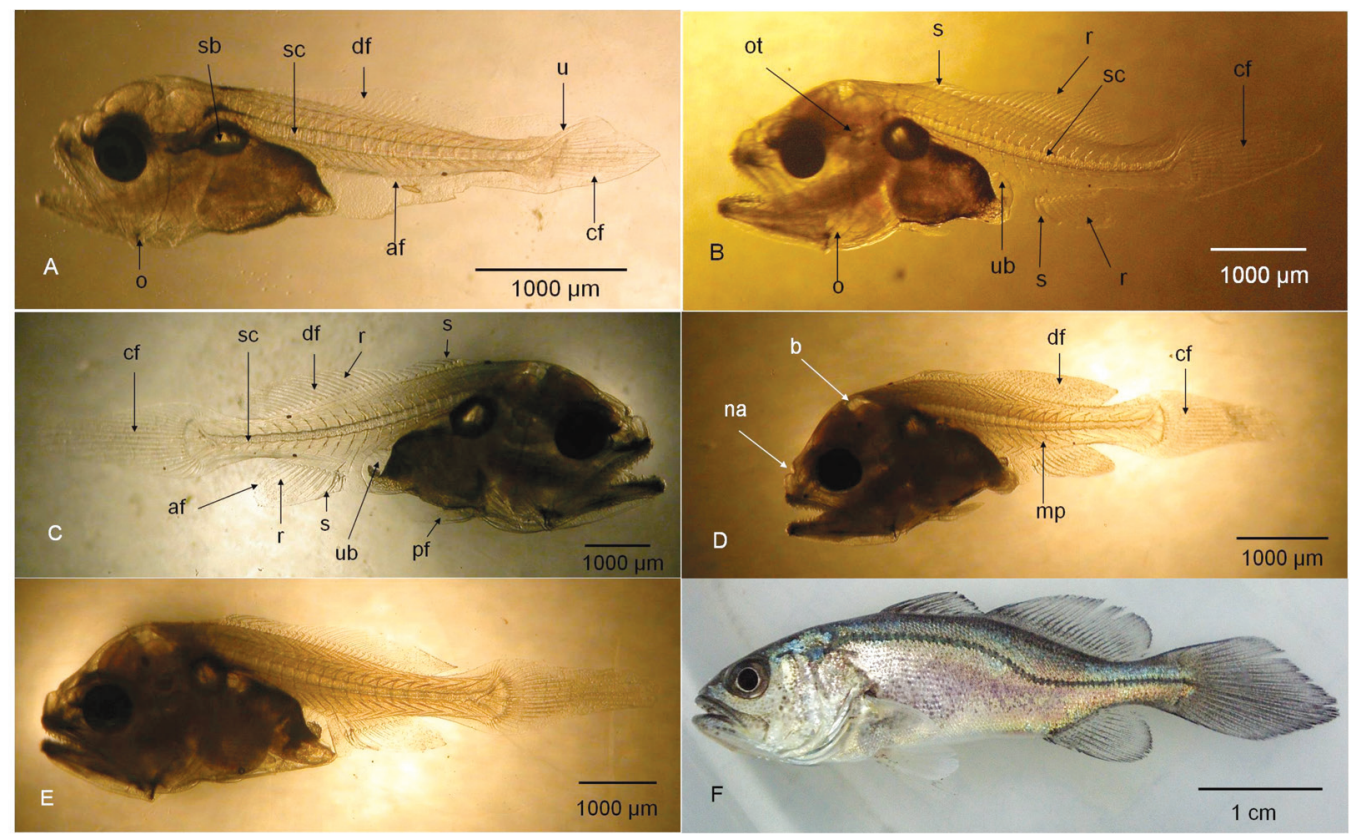

Fig. 4. Larval development of C. squamipinnis. (A) Postflexion larvae (14 das). (B) Postflexion larvae (20 das). (C) Postflexion larvae (22 das). (D) Postflexion larvae (25 das). (E) Juvenil (29 das). (F) Scaled juvenil (47 das). (sc) Spinal column. (s) Spine. (df) Dorsal fin. (u) Urostile. (cf) Caudal fin. (af) Anal fin. (ot) Otolith. (r) Radius. (ub) Urinary bladder. (o) Operculum. (pf) Pelvic fin. (na) Narina. (b) Brain. (mp) muscular package.

Fig. 4. Desarrollo larval de C. squamipinnis. (A) Larva en postflexión (14 ddd). (B) Larva postflexión (20 ddd). (C) Larva en postflexión (22 ddd). (D) Larva en postflexión (25 ddd). (E) Juvenil (29 ddd). (F) Juvenil escamado (47 ddd). (sc) Columna vertebral. (s) Espina. (df) Aleta dorsal. (u) Urostilo. (cf) Aleta caudal. (af) Aleta anal. (ot) Otolito. (r) Radios. (ub) Vejiga urinaria. (o) Opérculo. (pf) Aleta pélvica. (na) Narina. (b) Cerebro. (mp) Paquete muscular.

los radios, mientras que a los 25 ddd (7.401 $\pm 0.610 \mathrm{~mm}(\mathrm{LT}), 5.841 \pm 0.445 \mathrm{~mm}(\mathrm{LN})$, Fig. 4D), los músculos del tronco (mp) se definieron y dispusieron en forma de zigzag en posición dorsal y ventral, y a lo largo de todo el cuerpo como paquetes musculares. Por otro lado, se definieron las narinas (na), por encima de la boca y en frente de los ojos.

A los 29 ddd $(8.239 \pm 0.615 \mathrm{~mm}(\mathrm{LT})$ y $6.288 \pm 0.291 \mathrm{~mm}$ (LN), Fig. 4E), los radios y las espinas de todas las aletas estuvieron bien desarrollados, los músculos en forma de "v" se definieron, así como los opérculos y el cerebro. Se observó que hasta este día las larvas aún no iniciaron el proceso de escamado.

El proceso de escamado coincidió con el periodo de transición de alimento vivo a alimento inerte, que ocurrió entre los días 35 y 45 ddd. Las larvas iniciaron el escamado cerca de las aletas dorsal y anal, y sobre la línea lateral, y conforme pasaron los días, la larva se transformó en un adulto pequeño (Fig. 4F), con un peso promedio de $3.68 \pm 1.09 \mathrm{~g}$, un peso mínimo de $0.3 \mathrm{~g}$ y un peso máximo de 6.2 g. a los 47 ddd.

La LT promedio de las larvas se incrementó diariamente $(\mathrm{R} 2=0.974)$, siendo al momento de la eclosión de $2.234 \pm 0.122 \mathrm{~mm}$, hasta que alcanzó una LT de $8.239 \pm 0.615$ $\mathrm{mm}$ al final del experimento (Fig. 5A). La LN promedio fue de $2.179 \pm 0.119 \mathrm{~mm}$ (Promedio $\pm \mathrm{DE}$ ), mientras que después de 29 ddd fue de $6.288 \pm 0.291 \mathrm{~mm}$. Por otra parte, la LN siguió una tendencia similar a la que se observó para 

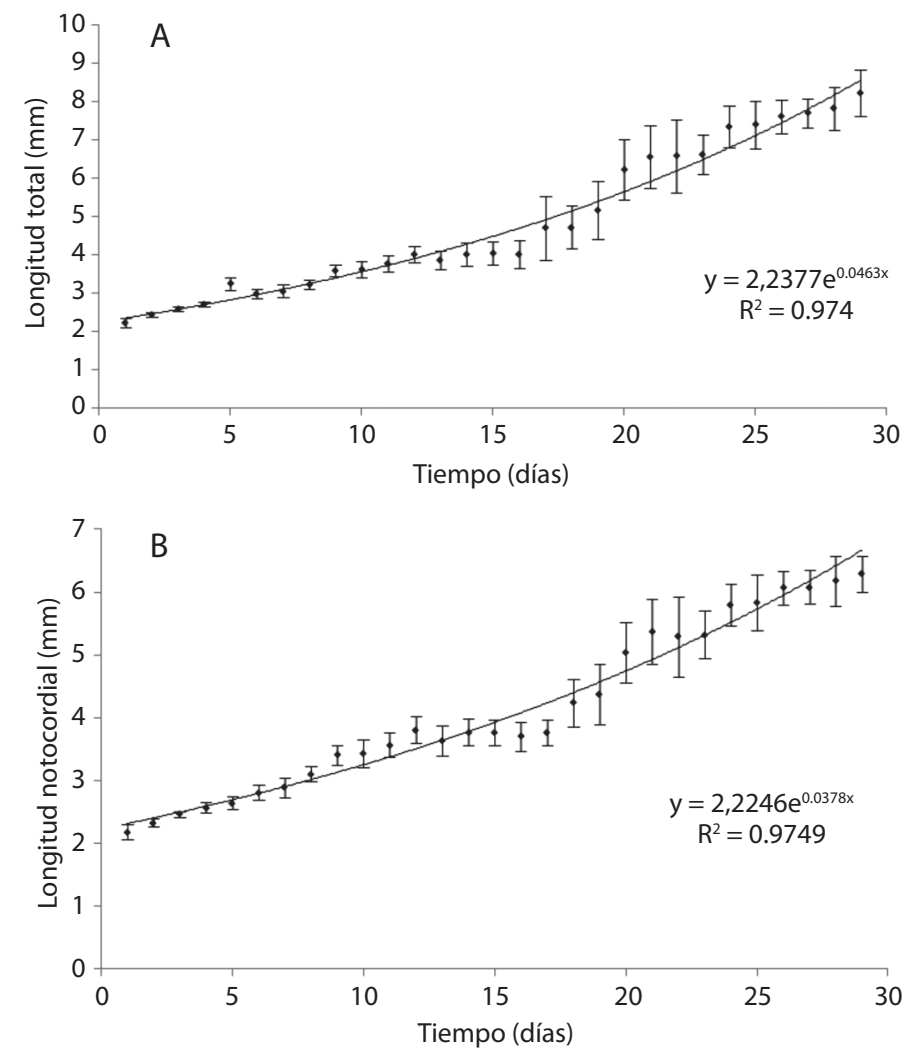

Fig. 5. Daily variation in mean total length (A) and mean notochordial length (B) of corvina aguada larvae (C. squamipinnis) during the laboratory rearing period.

Fig. 5. Variación diaria en la Longitud total promedio (A) y la Longitud notocordial promedio (B) de la corvina aguada (C. squamipinnis) durante el periodo de levantamiento en el laboratorio.

la LT, con una DE que se incrementó a través del tiempo (Fig. 5B, R2 = 0.9635).

\section{DISCUSIÓN}

En estudios donde se reporta la reproducción en cautiverio de las corvinas, indican que éstas alcanzan estados de madurez avanzados, vitelogénesis tardía y espermiación en cautiverio. Sin embargo, exhiben una disfunción reproductiva durante la vitelogénesis que no permite la ovulación y el desove espontáneamente, por lo que, es necesario la aplicación de terapias hormonales para que se complete dicho proceso (Duncan et al., 2012; Mylonas et al., 2013a; b). Según Cárdenas (2011), la única especie reportada con desoves espontáneos en cautiverio es el corvinón ocelado (S. ocellatus), y Mylonas et al. (2013a) reportaron por primera vez un desove espontáneo de la corvina mediterránea, A. regius, con fertilización superior al $97 \%$, siendo la posible causa una mejor aclimatación a las condiciones de cautiverio, en lugar de tres años de aclimatación, mantenerlos cuatro años. Por lo anterior, la corvina aguada, C. squamipinnis, sería una de las pocas especies de corvina que alcanza la madurez en cautiverio y desova espontáneamente, sin necesidad de la aplicación de tratamientos hormonales, produciendo en cautiverio huevos fértiles y juveniles como primera generación, bajo las condiciones reportadas en este estudio. En nuestro caso, las corvinas aguadas estuvieron en cautiverio desde octubre 2006 hasta enero 
2009, que fue cuando se dieron los desoves (2 años y 3 meses).

Los huevos fertilizados de la corvina aguada, C. squamipinnis, fueron transparentes, esféricos, totalmente lisos, pelágicos y no adheribles, con vitelo uniforme y un espacio perivitelino muy pequeño. Estas características han sido reportadas, en general, para los peces marinos (Blaxter, 1988). Cuartas et al. (2003) encontraron que los huevos del corocoro (Haemulon bonariense) fueron esféricos, pelágicos, con un corion transparente, sin proyecciones, totalmente liso, con vitelo uniforme y no coloreado.

En este estudio, el diámetro promedio de los huevos fue de $0.852 \pm 0.039 \mathrm{~mm}$, con un diámetro mínimo de $0.757 \mathrm{~mm}$ y el máximo de $0.920 \mathrm{~mm}$. El diámetro de los huevos de peces marinos puede medir cerca de $1 \mathrm{~mm}$, aunque se pueden encontrar desde los $0.6 \mathrm{~mm}$ hasta los $4 \mathrm{~mm}$, dependiendo de la especie (Blaxter, 1988; Ditty et al., 2006). En el caso de las corvinas, y específicamente en la corvina mediterránea, A. regius, Papadakis et al. (2013), reportaron un diámetro de $1.140 \pm 0.010 \mathrm{~mm}$, y Jiménez et al. (2007) midieron un diámetro promedio de $0.850 \pm 0.020 \mathrm{~mm}$, mientras que Gamsiz y Neke (2008) obtuvieron un diámetro promedio de $0.857 \pm 0.023 \mathrm{~mm}$, y un rango de $0.825 \mathrm{~mm}$ a $0.910 \mathrm{~mm}$. Para A. japonicus, el diámetro promedio fue de $0.839 \pm 0.150$ $\mathrm{mm}$ (Ballagh et al., 2011). En algunos casos se han observado diferencias en los diámetros en dependencia del tratamiento hormonal realizado a los reproductores de corvina. Al realizar implantes hormonales, el diámetro de los huevos obtenidos fue de $0.990 \pm 0.020 \mathrm{~mm}$; en contraste, los huevos obtenidos mediante inyecciones tuvieron un diámetro de $0.930 \pm$ $0.010 \mathrm{~mm}$ (Cárdenas, 2011). Estas diferencias en diámetros podrían deberse también a características propias de las especies, las condiciones de mantenimiento de los reproductores y de los huevos, y la precisión de los instrumentos utilizados para hacer las mediciones.

En este estudio, se observó que la mayoría de los huevos fertilizados poseían una sola gota oleosa, y en muy pocos, un máximo de tres gotas. Una característica distintiva de los huevos de peces marinos es la presencia de una gota de aceite (oleosa) (Boza-Abarca et al., 2008), o varias gotas pequeñas oleosas que se unen para formar una sola (Ditty et al., 2006). Jiménez et al. (2007), observaron en los huevos de la corvina mediterránea (A. regius), la presencia de una sola gota oleosa, mientras que Gamsiz y Neke (2008), para la misma especie reportaron de 2-4 gotas oleosas. En el verrugato del sur (A. japonicus), Ballagh et al. (2011) describieron que en muy pocos huevos se logra detectar hasta dos gotas. En otras especies marinas se ha observado una sola gota de aceite en los huevos fertilizados (Cuartas et al., 2003; Hernández et al., 2012).

El diámetro de la gota de aceite (gota oleosa) en los huevos de C. squamipinnis, midió $0.27 \pm 0.016 \mathrm{~mm}$ (Volumen de $0.240 \pm 0.025$ $\mathrm{mm} 3)$. Este valor es superior al reportado por Gamsiz y Neke (2008), para A. regius, cuyo diámetro fue de $0.215 \pm 0.010 \mathrm{~mm}$, pero es menor al reportado por Hernández et al. (2012) de $0.490 \pm 0.100 \mathrm{~mm}$, para la especie Eugerres mexicanus (Gerridae), cuyos huevos permanecen adheridos al fondo, y al reportado por Cuartas et al. (2003) en los huevos del corocoro (H. bonariense), con un diámetro promedio de $0.200 \pm 0.020 \mathrm{~mm}$.

En relación con el desarrollo embrionario, los tiempos de formación de las etapas embrionarias difirieron levemente a lo reportado para otras especies de corvinas. En este estudio, las primeras cinco divisiones celulares en los huevos fertilizados de C. squamipinnis ocurrieron 3 hdd, mientras que en la corvina $A$. regius los huevos llegaron a esta quinta división en 2:40 hdd (Gamsiz \& Neke, 2008), y en el verrugato del sur, A. japonicus, en 4 hdd (Ballagh et al., 2011). En otras especies de peces marinos, la quinta división celular ocurre 1:14 hdd $(H$. bonarienses). Aunque los tiempos para alcanzar esta quinta división son similares entre $C$. squamipinnis y $A$. regius, sí difieren del reportado para $A$. japonicus.

El tiempo de formación de la mórula, blástula y gástrula observados en la corvina $C$. squamipinnis, duran en total 5 horas (3:30-8:30 
hdd), similar al intervalo de tiempo que tardan en la corvina mediterránea (A. regius) (3:259:05 hdd) (Jiménez et al., 2007; Gamsiz \& Neke, 2008).

Las etapas finales del desarrollo embrionario de la corvina aguada (C. squamipinnis), fueron muy parecidas a las reportadas por Gamsiz y Neke (2008). En dicho estudio, la forma embrionaria de " $\mathrm{C}$ " de la corvina mediterránea (A. regius), se observó a las 10:10 hdd y la forma de "S" a las 23:30 hdd. En el caso del verrugato del sur (A. japonicus) (Ballagh et al., 2011), la forma "C" ocurre $11 \mathrm{hdd}$, y la forma " $\mathrm{S}$ " 25 hdd. En este estudio, la forma "C" ocurre a las 10:00 hdd, y la forma "S" se detectó dos horas después, cuando la cola se ha desprendido.

En la corvina aguada (C. squamipinnis), la eclosión del huevo ocurrió 19:00 hdd. La eclosión de las larvas de $A$. regius ocurre entre 23:30-24:00 hdd (Jiménez et al., 2007; Gamsiz \& Neke, 2008), mientras que para A. japonicus ocurre a las 28:00 hdd (Ballagh et al., 2011). Estas diferencias en el momento de la eclosión, se pueden deber a la temperatura de incubación de los huevos, ya que, tanto para la corvina mediterránea (A. regius) como para $A$. japonicus, los rangos de temperatura oscilaron entre $22-24{ }^{\circ} \mathrm{C}$ (Jiménez et al., 2007; Gamsiz \& Neke, 2008), lo que difiere mucho de la temperatura de incubación medida en este estudio (28 $\left.{ }^{\circ} \mathrm{C}\right)$. Para el caso de A. japonicus, su eclosión ocurre 28:00 hdd a una temperatura similar a la utilizada con $A$. regius $\left(22^{\circ} \mathrm{C}\right)$. En este caso, la diferencia parece ser más consecuencia genética de la especie.

En este estudio, las larvas de C. squamipinnis poseen la gota de aceite en posición media ventral y cerca del futuro ano. Esta posición de la gota de aceite coincide con la encontrada en la corvina mediterránea (Jiménez et al., 2007; Gamsiz \& Neke, 2008) y el verrugato del sur (Ballagh et al., 2011). En otras especies, como E. mexicanus y H. bonarienses (Cuartas et al., 2003; Hernández et al., 2012), la posición de la gota oleosa es anteroventral con respecto a la cabeza, al igual que en larvas de L. guttatus (Boza-Abarca et al., 2008). El nado de las larvas se ve afectado por esta posición de la gota de aceite, ya que, las larvas con la gota cercana a la cabeza tienden a tener un nado vertical (Boza-Abarca et al., 2008). En el caso de C. squamipinnis, el nado fue horizontal y cercano al fondo, con desplazamientos cortos.

Las larvas recién eclosionadas en este estudio presentaron un valor de LT promedio de $2.234 \pm 0.122 \mathrm{~mm}$, que corresponde al menor valor de LT reportado en las especies de corvinas estudiadas, y no se compara con el $4.020 \pm 0.260 \mathrm{~mm}$ de LT en larvas de otras especies como E. mexicanus (Hernández et al., 2012). Las larvas recién eclosionadas del verrugato del Sur (A. japonicus) variaron en su LT, Ballagh et al. (2011) obtuvieron un valor de $2.3 \mathrm{~mm}$, mientras que Musson y Kaiser (2014) de $2.56 \pm 0.09 \mathrm{~mm}$ (promedio $\pm \mathrm{DE}, \mathrm{n}=150$ ). En la corvina mediterránea (A. regius), también se han reportado diferencias, Jiménez et al. (2007) midieron una LT de $2.82 \pm 0.37 \mathrm{~mm}$, y Gamsiz y Neke (2008) de $2.593 \pm 0.055$ mm. Esto es normal y puede atribuirse a diversos factores que afectan la calidad del huevo y de la larva, entre los cuales se destacan la nutrición y el estado de los reproductores, el ciclo de desoves y los factores genéticos propios de la especie (Avilés, 2005; Pepe-Victoriano, Araya, \& Faúndez, 2012).

Es importante destacar que para el momento de la eclosión, la única aleta presente fue la aleta embrionaria (ef). Esto concuerda con lo reportado para el verrugate (U. cirrosa) (Koumoundouros et al., 2005).

El tiempo de absorción del saco vitelino (alimentación endógena) depende de la especie (Borçato, Bazzoli, \& Sato, 2004), del tamaño del huevo, y de la temperatura (Blaxter, 1988). En este estudio, el tiempo de absorción del saco vitelino ( $1.5 \mathrm{ddd})$ de la corvina aguada $(C$. squamipinnis), fue menor al observado para el verrugato del sur (A. japonicus) (Musson \& Kaiser, 2014) y la corvina mediterránea $(A$. regius) (Gamsiz \& Neke, 2008), el cual fue 3 ddd en ambas especies.

La alimentación exógena (preflexión), de la corvina aguada inició 49 hdd (2.04 ddd), esto ocurrió de manera paralela con la apertura 
de la boca y la pigmentación de los ojos. Este tiempo es menor a los 3 ddd que tarda $A$. regius (Gamsiz \& Neke, 2008), o a los 4-6 ddd, reportados para el verrugato del sur (A. japonicus) (Musson \& Kaiser, 2014), pero similar a las 48 hdd en $H$. bonarienses, especie perteneciente a la familia Haemulidae (Cuartas et al., 2003).

En este estudio, las zonas de pigmentación se observaron 2 ddd. Al mismo tiempo, se observó la aparición de otras estructuras, como los otolitos y el tracto digestivo en formación. La literatura reporta que en las corvinas, es común que, tanto la gota de aceite como el embrión, estén pigmentados. Además, la utilización de patrones de pigmentación para identificar huevos y larvas en proceso de absorber el saco vitelino, ya ha sido investigada y aplicado como un método para identificar especies. Sin embargo, esta forma de identificación tiene una limitante muy grande, debido a que los cromatóforos se deterioran rápidamente cuando la larva muere y se preserva (Ditty et al., 2006). Los tiempos en los cuales aparecieron las zonas de pigmentación y otras estructuras son similares a lo reportado para la corvina mediterránea (A. regius), ocurriendo la aparición de los otolitos y el tracto digestivo 3 dde, cuando la larva tenía una longitud total de $2.930 \pm 0.130 \mathrm{~mm}$ (Jiménez et al., 2007).

En la corvina aguada (C. squamipinnis), la vejiga natatoria se observó $4 \mathrm{ddd}$, a una LT menor $(2.705 \pm 0.069 \mathrm{~mm})$ similar a la corvina mediterránea (A. regius) a los $4 \mathrm{ddd}$, cuando la larva tuvo una LT de $2.780 \pm 0.270 \mathrm{~mm}$ (Jiménez et al., 2007).

En este estudio, la flexión en la corvina aguada ocurrió a los 11 ddd (LT: $3.767 \pm 0.209$ $\mathrm{mm}$ ), con un engrosamiento notable del notocordio y a su vez, con la aparición de la vejiga urinaria (ub) a los 13 ddd (LT: $3.860 \pm 0.253$ $\mathrm{mm})$. En contraste, en el estudio realizado por Gamsiz y Neke (2008), la flexión en las larvas de la corvina mediterránea (A. regius), se observó 15 ddd, mientras que Jiménez et al. (2007) lo reportó 12 ddd (LT: $3.970 \pm 0.260$ $\mathrm{mm}$ ). En otra especie marina (E. mexicanus), la flexión ocurrió 16 dde (Hernández et al.,
2012). En relación a la vejiga urinaria, estudios realizados para determinar el desarrollo del tracto digestivo en larvas del verrugato del sur (A. japonicus), también reportan su aparición entre los días 8 y 12 (Musson \& Kaiser, 2014).

La postflexión en las larvas de la corvina aguada se observó a los 14 ddd (LT: $4.015 \pm$ $0.302 \mathrm{~mm}$ ), junto con la formación del urostilo (u). En contraste, la postflexión en la corvina mediterránea ocurre a los $13 \mathrm{ddd}$, con una LT de $4.330 \pm 0.430 \mathrm{~mm}$ (Jiménez et al., 2007), mientras que en la especie E. mexicanus, la postflexión se observó 24 dde con una LT de $10.330 \pm 1.450 \mathrm{~mm}$, superior en tiempo y tamaño con respecto a todas las corvinas.

La postflexión en la corvina aguada $(C$. squamipinnis), también estuvo marcada por la aparición de la columna vertebral (sc) y la definición morfológica de las aletas caudal (cf) y anal (af) con sus radios ya visibles (14 ddd). Por su parte, en A. nobilis la aparición de los radios que definen la aleta caudal y anal se dio 15 ddd y 22 ddd, respectivamente (Moser, Ambrose, Busby, Butler, \& Sandknop, 1983).

En este estudio, los opérculos se terminaron de desarrollar a los $22 \mathrm{ddd}$, similar a lo reportado por Jiménez et al. (2007), en larvas de A. regius a los $23 \mathrm{ddd}$, con una LT de 7.070 $\pm 0.620 \mathrm{~mm}$. Al día $25 \mathrm{ddd}$, los paquetes musculares son fácilmente distinguibles en la corvina aguada, lo que coincide con lo observado en la corvina mediterránea. Los radios y las espinas de todas las aletas se encuentran completamente formados en la corvina aguada a los 29 ddd (LT: $8.239 \pm 0.615 \mathrm{~mm}$ ), este cambio fue reportado por Jiménez et al. (2007) a los 30 ddd, a una LT de $11.660 \pm 0.960 \mathrm{~mm}$.

En conclusión, este estudio demostró que la corvina aguada se puede reproducir espontáneamente en cautiverio espontáneamente, que las larvas producidas pueden criarse utilizando las técnicas básicas en el alevinaje de peces marinos, y que se pueden producir juveniles para ser utilizados con fines de maricultura.

\section{AGRADECIMIENTOS}


Este estudio fue financiado con la Ley del Atún, $N^{\circ}$ 6267, y la Escuela de Ciencias Biológicas de la Universidad Nacional de Costa Rica. Los autores agradecen al Capitán Orlando Torres por recolectar y transportar los peces de las jaulas a la Estación de Biología Marina Juan Bertoglia Richards. Deseamos agradecer la revisión detallada al manuscrito realizada por los revisores anónimos, lo cual contribuyó a enriquecer la información presentada.

\section{RESUMEN}

Las corvinas son especies de interés comercial que han sido sobreexplotadas en el Golfo de Nicoya, Costa Rica. El objetivo de este estudio fue describir por primera vez, el desove espontáneo y la ontogenia de las larvas de la corvina aguada Cynoscion squamipinnis en cautiverio, con el fin de realizar repoblamiento y proyectos de maricultura. Reproductores silvestres ( $n=6,1-2 \mathrm{Kg}$ ), fueron capturados y mantenidos en la Estación de Biología Marina Juan Bertoglia Richards (Puntarenas, Costa Rica) por un periodo de dos años (octubre 2006-diciembre 2008). Durante este periodo, el estado de madurez de las hembras $(n=3)$ fue registrado mediante muestras de cánula y los machos $(n=3)$ mediante masaje abdominal. Todos los reproductores fueron colocados juntos en un tanque de $18 \mathrm{t}$, con aireación, a una salinidad entre 33-35 ups y expuestos a una temperatura constante $\left(29 \pm 1{ }^{\circ} \mathrm{C}\right)$. De enero a marzo 2009 ocurrió un periodo de desove espontáneo, obteniéndose 162000 huevos en tres desoves. El porcentaje de fertilización fue de 50-60\%, y la supervivencia después de la eclosión fue entre 60-85 \%. El diámetro de los huevos fue de $0.852 \mathrm{~mm}$ (Desviación Estándar=0.039), con una gota de aceite de $0.269 \mathrm{~mm}(\mathrm{DE}=0.016)$. En el desarrollo embrionario, la primera división mitótica ocurrió una hora después del desove (hdd), la segunda división 1:30 hdd, la tercera división 2 hdd, la cuarta división a las 2:30 hdd, y la quinta 3:00 hdd. La mórula fue observada a las 3:30 hdd, la blástula a las 4:30 hdd, la gástrula a las 8:30 hdd, la forma $\mathrm{C}$ a las 10:00 hdd, y la forma $\mathrm{S}$ ocurrió de las 10-19 hdd. La eclosión ocurrió 19 hdd. La larva midió $2.234 \mathrm{~mm}$ $(\mathrm{DE}=0.122)$ de longitud total $(\mathrm{LT})$, y $2.179 \mathrm{~mm}(\mathrm{DE}=$ 0.119 ) de longitud notocordial (LN). La preflexión inició 49 hdd, la flexión 11 días después del desove (ddd) (3.767 $\mathrm{mm} \mathrm{LT}, \mathrm{DE}=0.209)$, y la postflexión a los $14 \mathrm{ddd}(4.015$ $\mathrm{mm} \mathrm{LT}, \mathrm{DE}=0.302)$. A los $45 \mathrm{ddd}$, los juveniles pesaron $3.68 \mathrm{~g}(\mathrm{DE}=1.09)$. El tiempo de eclosión de las larvas de la corvina aguada fue menor al de otras especies de corvina. Los tiempos de formación en las etapas embrionarias difirieron poco con respecto a los tiempos observados para otras especies de corvinas. Las diferencias observadas con respecto a otras especies, probablemente responden a las características genéticas propias de cada especie y a la temperatura de incubación de los huevos. La obtención de desoves espontáneos sin aplicaciones hormonales a los reproductores, y la producción de juveniles en cautiverio demostraron que la corvina aguada puede ser considerada en programas de repoblamiento y proyectos de maricultura.

Palabras clave: Cynoscion squamipinnis, corvina aguada, reproducción, ontogenia, desarrollo larval.

\section{REFERENCIAS}

Avilés, M. (2005). Calidad de huevos y larvas según el manejo de los reproductores de la Cabrilla (Paralabrax maculatofasciatus, Pisces: Serranidae) (Tesis de Doctorado). Universidad de Barcelona, Barcelona, España.

Ballagh, A. D., Pankhurst, P. M., \& Fielder, D. S. (2011). Embryonic development of mulloway, Argyrosomus japonicus, and egg surface disinfection using ozone. Aquaculture, 318, 475-478.

Blaxter, J. (1988). Pattern and variety in development. En W. Hoar, \& D. Randall (Eds.), Fish Physiology (pp. 1-58). Parte A. Nueva York: Academic Press.

Borçato, L. F., Bazzoli, N., \& Sato, Y. (2004). Embriogenesis and larval ontogeny of the 'pia-gordura', Leporinus piau (Fowler) (Pisces, Anastomidae) after induced spawning. Revista Brasilera de Zoología, 21, 117-122.

Botta, P., Sciara, A., Arranz, S., Murgas, L. D. S., Pereira, G. J. M., \& Oberlender, G. (2010). Estudio del desarrollo del sábalo (Prochilodus lineatus). Archivos de Medicina Veterinaria, 42, 109-114.

Boza-Abarca, J., Calvo-Vargas, E., Solís-Ortiz, N., \& Komen, J. (2008). Desove inducido y crecimiento larval del pargo machado, Lutjanus guttatus, en la Estación de Biología Marina de Puntarenas, Costa Rica. Ciencias Marinas, 34, 239-252.

Cárdenas, S. (2011). Cultivo de corvina (Argyrosomus regius). Cuadernos de acuicultura. Madrid: Fundación Observatorio Español de Acuicultura, Madrid, España.

Cárdenas, S. (2012). Biología y acuicultura de corvinas en el mundo. AquaTIC, 37, 1-13.

Chacón, A., Araya, H., Vásquez, A., Brenes, R., Marín, B., Palacios, J., Soto, R., Mejía-Arana, F., Shimazu, Y., \& Hiramatsu, K. (2007). Estadísticas Pesqueras del Golfo de Nicoya, Costa Rica, 19942005. Proyecto Manejo Sostenible de la Pesquería para el Golfo de Nicoya. Puntarenas, Costa Rica: JICA-UNA-INCOPESCA. 
Cuartas, A., Rosas, J., Velásquez, A., \& Cabrera, T. (2003). Inducción al desove, Desarrollo embrionario, y larval de corocoro rayao Haemulon bonariense Cuvier, 1830 (Pisces: Haemulidae). Revista de Biología Marina y Oceanografia, 38, 27-37.

Ditty, G. J., Shaw, R. F., \& Farooqui, T. (2006). Sciaenidae. En W. Richards (Ed.), Early stages of Atlantic Fishes (pp. 1-15). Florida, USA: Taylor \& Francis Group.

Duncan, N., Estévez, A., Porta, J., Carazo, I., Norambuena, F., Aguilera, C., Gairin, I., \& Bucci, F. (2012). Reproductive development, GnRHa-induced spawning and egg quality of wild meagre (Argyrosomus regius) acclimatised to captivity. Fish Physiology and Biochemistry, 38, 1273-1286.

FAO. (2012). El estado mundial de la pesca y acuicultura. Roma, Italia: Departamento de Pesca y Acuicultura FAO.

Fielder, D., Bardsley, W., Allan, G., \& Pankhurst, P. (2005). The effects of salinity and temperature on growth and survival of Australian snapper, Pagrus auratus larvae. Aquaculture, 250, 201-214.

Gamzis, K., \& Neke, M. (2008). Embryonic development stages of meagre Argyrosomus regius Asso 1801 under rearing conditions. $8^{\text {th }}$ Larval Biology Symposium. Julio, Lisboa, Portugal.

García-Alonso, J., \& Vizziano, D. (2004). Induction of oocyte maturation in the White croaker Micropogonias furnieri (Pisces: Sciaenidae) by human Chorionic gonadotropin. Brazilian Journal of Biology, $64,73-80$.

Hazmadi, M., Amin, S. M. N., Aminur, R. M., Hatta, M. M., Christianus, A., Siraj, S. S., \& Arshad, A. (2013). Embryonic and larval development of endangered Temoleh, Probarbus jullieni (Sauvage). Asian Journal of Veterinary Advances, 8, 362-368.

Hernández, E., Perera, M. A., Castillo, A., \& Luna, E. (2012). Embrionic and larval delopment of Eugerres mexicanus (Perciformes: Guerridae) in Tenosique, Tabasco, Mexico. Revista Biología Tropical, 60, 369-379.

Ignatius, B., Rathore, G., Jagadis, I., Kandasami, D., \& Victor, A. C. C. (2001). Spawning and larval rearing for tropical clown fish Amphiprion sebae under captive condition. Journal Aquaculture in the Tropics, 16, 241-249.
Jiménez, M. T., Pastor, E., Grau, A., Alconchel, J. I., Sánches, R., \& Cárdenas, S. (2005). Revisión del cultivo de esciénidos en el mundo, con especial atención a la corvina Argyrosomus regius (Asso, 1801). Boletín Instituto Español de Oceanografia, 21, 169-175.

Jiménez, M. T., Rodrígues, A., de La Rúa, A., Sánches, R., \& Cárdenas, S. (2007). Atlas de desarrollo de la corvina Argyrosomus regius (Pisces: Sciaenidae) durante su primer mes de vida. Revista Electronica Veterinaria, 8, 36.

Koumoundouros, G., Kouttouki, S., Georgakopoulou, E., Papadakis, I., Maingot, E., Kaspiris, E., Kiriakou, Y., Georgiou, G., Divanach, P., Kentouri, M., \& Mylonas, C. (2005). Ontogeny of the shi drum Umbrina cirrosa (Linnaeus 1758), a candidate new species for aquaculture. Aquaculture Research, 36, 1265-1272.

Moretti, A., Fernández, M., Cittolin, G., \& Guidastri, R. (1999). Manual on Hatchery Production of Seabass and Gilthead Seabream. Rome, Italy: FAO.

Moser, H. G., Ambrose, D. A., Busby, M., Butler, J., \& Sandknop, E. (1983). Description of early stages of white seabass, Atractoscion nobilis, with notes on distribution. California Cooperative Oceanic Fisheries Investigations, 24, 182-193.

Musson, M., \& Kaiser, H. (2014). Development of the digestive system in dusky kob, Argyrosomus japonicus. Aquac. International, 22, 783-796.

Mylonas, C. C., Mitrizakis, N., Papadaki, M., \& Sigelaki, I. (2013a). Reproduction of hatchery-produced meagre Argyrosomus regius in captivity I. Description of the annual reproductive cycle. Aquaculture, 414-415, 309-317.

Mylonas, C. C., Mitrizakis, N., Castaldo, C. A., Cerviño, C. P., Papadaki, M., \& Sigelaki, I. (2013b). Reproduction of hatchery-produced meagre Argyrosomus regius in captivity II. Hormonal induction of spawning and monitoring of spawning kinetics, egg production and egg quality. Aquaculture, 414-415, 318-327.

Olivares, O. P., \& Boza, J. A. (1999). Crecimiento de juveniles de pargo mancha (Lutjanus guttatus) utilizando alimento granulado en condiciones de laboratorio. Uniciencia, 16, 45-48.

Otárola, R. (2008). Producción acuícola. Revista Ambientico, 179, 3-6.

Papadakis, I. E., Kentouri, M., Divanach, P., \& Mylonas, C. (2013). Ontogeny of the digestive system of meagre 
Argyrosomus regius reared in a mesocosm, and quantitative changes of lipids in the liver from hatching to juvenile. Aquaculture, 388-391, 78-88.

Pepe-Victoriano, R., Araya, M., \& Faúndez, V. (2012). Efecto de la temperatura en la supervivencia y primeros estadios larvales de Psetta maxima. International Journal of Morphology, 30, 1551-1557.

Ramírez-Merlano, J. A., Otero-Paternina, A. M., CorredorSantamaría, W., Medina-Robles, V. M., Cruz-Casallas, P. E., \& Velasco-Santamaría, Y. M. (2010). Utilización de organismos vivos como primera alimentación de larvas de yaque (Leiarius marmoratus) bajo condiciones de laboratorio. Orinoquia, 14, 45-58.

Thomas, P., Arnold, C. R., \& Holt, G. (1995). Red Drum and other Sciaenids. En N. R. Bromage, \& R. J. Roberts (Eds.), Broodstock management and egg and larval quality (pp. 118-137). Great Britain: Blackwell Science.

Zavala-Leal, I., Dumas-Lampage, S., \& Peña-Martínez, R. (2011). Organogénesis durante el periodo larval en peces. Oceanides, 26, 19-30. 
